\title{
Computational homogenization of diffusion in three-phase mesoscale concrete
}

\author{
Filip Nilenius ${ }^{1,2}$, Fredrik Larsson ${ }^{2}$, Karin Lundgren ${ }^{1}$, and \\ Kenneth Runesson ${ }^{2}$ \\ ${ }^{1}$ Department of Civil and Environmental Engineering \\ 2 Department of Applied Mechanics, \\ Chalmers University of Technology, Göteborg, Sweden
}

\begin{abstract}
A three dimensional (3D) mesoscale model of concrete is presented and employed for computational homogenization in the context of mass diffusion. The mesoscale constituents of cement paste, aggregates and Interfacial Transition Zone (ITZ) are contained within a Statistical Volume Element (SVE) on which homogenization is carried out. The model implementation accounts for ITZ anisotropy thereby the diffusivity tensor depends on the normal of the aggregate surface. The homogenized response is compared between $3 \mathrm{D}$ and $2 \mathrm{D}$ SVEs to study the influence of the third spatial dimension, and for varying mesoscale compositions to study the influence of aggregate content on concrete diffusivity. The computational results show that the effective diffusivity of 3D SVEs is about $40 \%$ greater than 2D SVEs when ITZ is excluded for the SVE, and $17 \%$ when ITZ is included. The results are in agreement with the upper Hashin-Shtrikman bound when ITZ is excluded, and close the Taylor assumption when ITZ is included.
\end{abstract}




\section{Introduction}

Chloride ions can substantially reduce the bearing capacity of concrete structures, such as bridges and offshore constructions, if the ions come in contact with the reinforcement bars and initiate corrosion. The source of chloride ions is often de-icing salts put on roads in winter times, or dissolved salt in seawater. Corrosion due to chloride ions is, thus, an important aspect of the durability of concrete structures since it is the the cause of time and resource consuming maintenance work.

The ingress rate of chloride ions, in turn, is highly dependent on the diffusion properties of concrete. Hence, if the diffusion properties of concrete can be modelled and estimated, a better prediction of the time span until corrosion initiation will be possible. Additionally, if those properties of concrete that affects the diffusivity the most can be determined, appropriate measures during concrete production can be taken so that the diffusivity of the material is reduced and thus making concrete structures better withstand exposure of chloride ions.

Computational homogenization $(\mathrm{CH})$ is an established technique to account for material heterogeneities at multiple length scales, while keeping computational costs at a moderate level, see e.g. Yuan and Fish [31], Geers et al. [8] and Miehe et al. [19]. One benefit of this technique is that it enables bulk properties of a material to be determine a priori in the case of linear subscale properties. This is beneficial if the macroscopic behaviour of a material is sought and the subscale features are highly complex. In case of non-linear material behaviour the subscale response needs to be determined concurrently during the computations in a nested fashion using a $\mathrm{FE}^{2}$-approach, as was done in Özdemir et al. [24] for transient heat problems and Larsson et al. [16] for problems of uncoupled consolidation of porous media using variationally consistent homogenization.

Concrete has a highly heterogeneous and random material structure at multiple length scales. Thus, considerable computational work on the effects of its heterogeneity has been done for this material: Kim and Al-rub [15] studied how the mesoscale phases of concrete contributes to the damage response of concrete using both 2D and 3D models, lattice based models were used Wang and Ueda [28] to simulate capillary adsorption in heterogeneous concrete and by Schlangen and Mier [25] to simulate fracture processes, which also was done by Carol et al. [6] using zero thickness interface elements as potential crack patterns. Hain and Wriggers $[10,11]$ used three dimensional computer tomography of hardened cement paste to compute effective material properties related to elasticity and damage.

On the cement paste level where the porosity is of importance, considerable work has been done with respect to diffusivity properties: Promentilla et al. [23], Bentz et al. [3] and Karim and Krabbenhoft [14] all used X-ray scanning in conjunction with techniques of random walk and Brownian motion to determine effective diffusivity properties of cement paste.

However, most work on numerical homogenization of concrete on the mesoscale reported in the literature treats mechanical problems. Diffusion processes in concrete 
greatly influence material properties and this field needs further exploration. A natural evolution in the development of $\mathrm{CH}$ is thus to study mass transport and at the same time account for all three spatial dimensions to obtain numerical results that potentially better reflect the real material behaviour.

This paper presents a 3D heterogeneous mesoscale model of concrete and compares numerical homogenization between 3D and 2D models to study how the third spatial dimension contributes to the homogenized results. This is done in the context of mass diffusion; thus, the effective diffusivity of concrete is the studied material property.

\section{Mesoscale SVE}

The concept of a Statistical Volume Element (SVE) is fundamental to $\mathrm{CH}$ techniques as it is the foundation on which all computational work is built. The SVE is expected to contain all material heterogeneities in a statistical manner and tends to become a Representative Volume Element (RVE) only when it is sufficiently large, see OstojaStarzewski [22]. The advantages of a numerical SVE, in conjunction with $\mathrm{CH}$, as opposed to e.g. analytical models are several:

- possible to account for complex sieve curves,

- possible to account for non-linear subscale material properties in a straight forward manner by use of $\mathrm{FE}^{2}$ methods,

- possible to study local material effects during transient conditions,

- possible to account for statistical spread in results by generating several SVEs of random topology.

In this work, the mesoscale material constituents are the cement paste matrix, aggregates and Interfacial Transition Zone (ITZ). The aggregates are modelled as spheres with random spatial distribution, enfolded by an interface layer of ITZ. The cement paste matrix is considered homogeneous, i.e. cracks are not accounted for herein.

\subsection{Sieve curve}

The aggregates in the SVE can be of arbitrary size and quantity. The volume fraction of each phase is defined as

$$
n_{\mathrm{a}}=\frac{V_{\mathrm{a}}}{V_{\mathrm{SVE}}}, \quad n_{\mathrm{cp}}=\frac{V_{\mathrm{cp}}}{V_{\mathrm{SVE}}}, \quad n_{\mathrm{ITZ}}=\frac{V_{\mathrm{ITZ}}}{V_{\mathrm{SVE}}},
$$

where $V_{\bullet}$ denotes volume in units of $\mathrm{cm}^{3}$ and sub-indices 'a' denotes aggregate and 'cp' denotes cement paste, respectively. Hence,

$$
V_{\mathrm{SVE}} \stackrel{\text { def }}{=} V_{\mathrm{a}}+V_{\mathrm{cp}}+V_{\mathrm{ITZ}} \text {. }
$$


The SVE model implementation allows for any realistic $n_{\mathrm{a}}$ to be obtained. Any sieve curve is possible to have as input for SVE realizations and we have the requirement that

$$
n_{\mathrm{a}}=n_{\mathrm{a}} \sum_{i=1}^{N} f_{i}, \quad \sum_{i=1}^{N} f_{i} \stackrel{\text { def }}{=} 1,
$$

where $f_{i}$ denotes the partial fraction of $n_{\mathrm{a}}$ for an aggregate size with radius $r_{i}$ in units of $\mathrm{cm}$, and $N$ denotes the total number of particles sizes included in the specified sieve curve. Both $N$ and $f_{i}$ can be chosen freely given the constraint in Eq. (3).

\subsection{Algorithm to generate SVEs}

The algorithm to generate SVEs is given below as pseudo-code. Figure 1 shows four examples of SVE realizations generated by the algorithm.

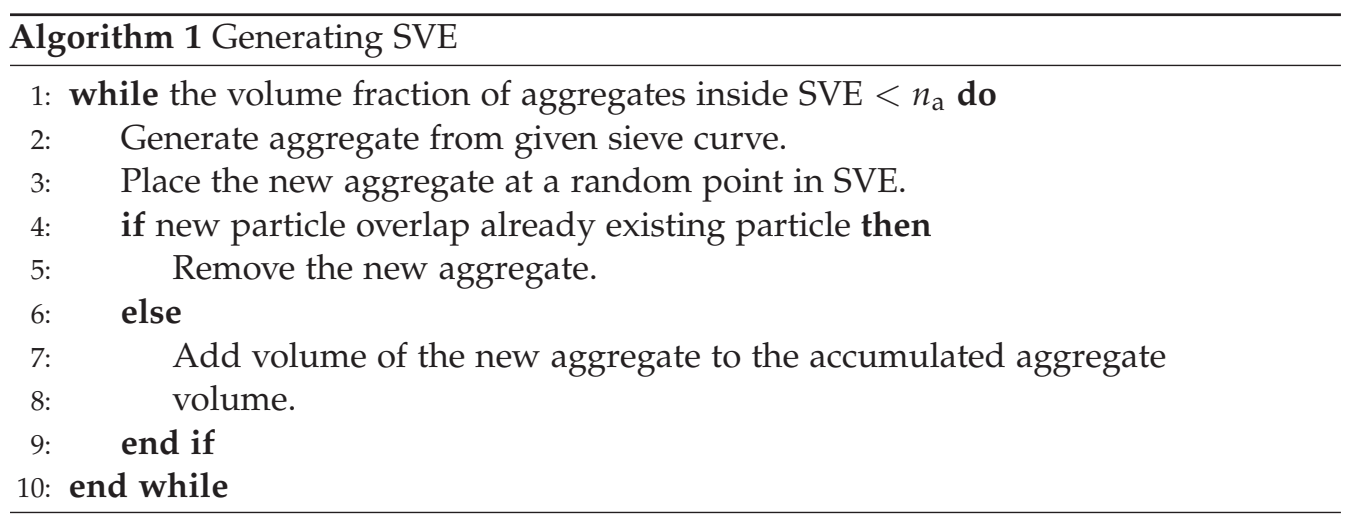

\subsection{Constitutive relations for the mesoscale materials}

We will use a constitutive relation for the mesoscale constituents on the form

$$
J=-\boldsymbol{D} \cdot \nabla \phi,
$$

where $J$ is the mass flux $\left[\mathrm{g} \mathrm{cm}^{-2} \mathrm{~s}^{-1}\right], \boldsymbol{D}$ is the second order diffusivity tensor $\left[\mathrm{cm}^{2} \mathrm{~s}^{-1}\right]$, $\nabla$ is the spatial gradient operator $\left[\mathrm{cm}^{-1}\right]$ and $\phi$ is mass concentration $\left[\mathrm{g} \mathrm{cm}^{-3}\right]$. The diffusivity tensors are given for each material as

$$
\boldsymbol{D}_{\mathrm{cp}}=D_{\mathrm{cp}} \boldsymbol{I}, \quad \boldsymbol{D}_{\mathrm{a}}=D_{\mathrm{a}} \boldsymbol{I}
$$

where $\boldsymbol{I}$ is the unit tensor and $\boldsymbol{D}_{\text {ITZ }}$ is defined in Section 4. 


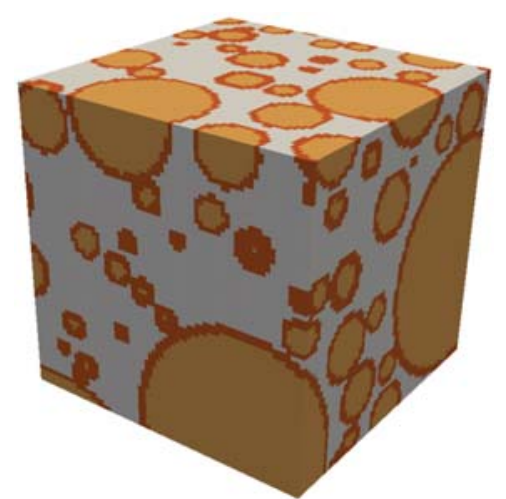

(a) $L_{\square}=2 \mathrm{~cm}$.

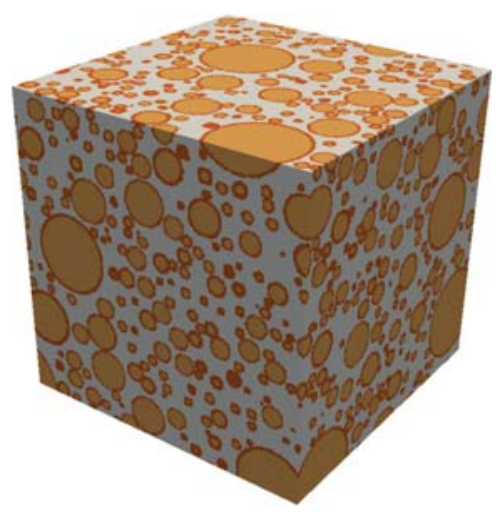

(c) $L_{\square}=6 \mathrm{~cm}$.

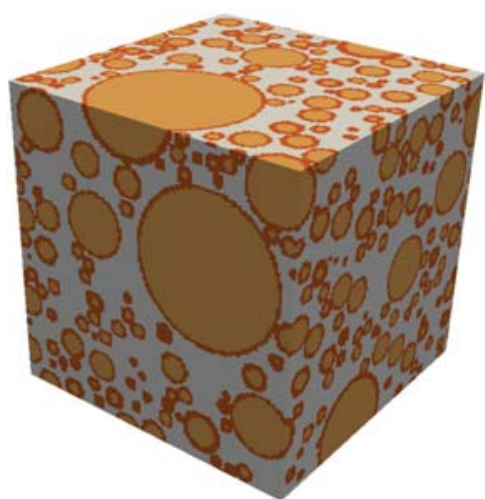

(b) $L_{\square}=4 \mathrm{~cm}$.

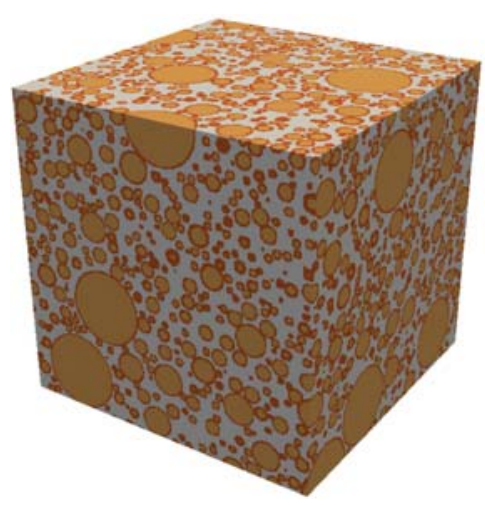

(d) $L_{\square}=10 \mathrm{~cm}$.

Figure 1: SVE realizations all having the same sieve curve with $n_{\mathrm{a}} \approx 0.42 . L_{\square}$ refers to the side length $[\mathrm{cm}]$ of the $S V E$. 


\section{FE discretization of SVE}

The spatial discretization technique is based on voxelization to create a structured grid of equally sized voxels (cubes). The concept is to subdivide the continuous SVE into a discrete set of voxels which are considered solid finite elements. This approach was used by Bentz et al. [2] and Garboczi [7] in the context of analytical homogenization of heterogeneous concrete and later adapted by Hain and Wriggers $[10,11]$ for elasticity problems and $\mathrm{CH}$. Other discretization approaches for 3D domains have been devised in the literature: both Caballero et al. [5] and Asahina and Bolander [1] used Voronoi tessellation where the aggregates were based on a polyhedron representation to generate 3D SVEs.

Material properties of the finite elements are determined by looping over all voxels/elements and for each element measure its distance to the center of all aggregates (spheres). If the distance is greater that the radius of any of the aggregates then the elements is located in the cement paste matrix and can be assigned its proper material property. If the distance is smaller than any aggregate radius then the element sits inside an aggregate.

\section{ITZ implementation}

Experimental results by Hedenblad [13] show that the effective diffusivity of concrete slightly increases with increasing aggregate content, alternatively remains unchanged. This implies that the Interfacial Transition Zone (ITZ) has a non-negligible effect on concrete diffusivity. The ITZ is a thin layer of cement paste which encapsulates the aggregates and has a much higher diffusivity than the remaining cement paste due to its high porosity, see e.g. Ollivier et al. [21] and Yang and Su [30]. The porosity is not constant within the ITZ but varies with a gradient and is highest closest to the surface of the aggregate. In effect, the transition from cement paste to ITZ is smooth and gradual. The porosity gradient is of importance since it constitutes the ITZ and, thus, enhance the diffusivity. For instance, Lutz et al [17] modelled this porosity gradient, within an elasticity context, by use of an power-law. However, to account for this gradient is outside the scope of this work and we will assume the gradient to be zero within the ITZ, but the porosity to be higher than in the cement paste.

As the SVEs are discretisized into a structured grid, a certain number of voxels end up at the interface between the cement paste matrix and aggregate, see Figure 2.

Consequently, these interface voxels will contain all three mesoscale materials: cement paste, aggregate and ITZ. For these voxels, we employ an anisotropic Voigt assumption on the over-all material diffusivity, expressed as

$$
\boldsymbol{D}_{\mathrm{ITZ}}=\frac{V_{\mathrm{a}} D_{\mathrm{a}}+V_{\mathrm{cp}} D_{\mathrm{cp}}}{h^{3}} \boldsymbol{I}+\frac{A_{\mathrm{ITZ}} D_{\mathrm{ITZ}} t}{h^{3}}(\boldsymbol{I}-\boldsymbol{n} \otimes \boldsymbol{n}),
$$




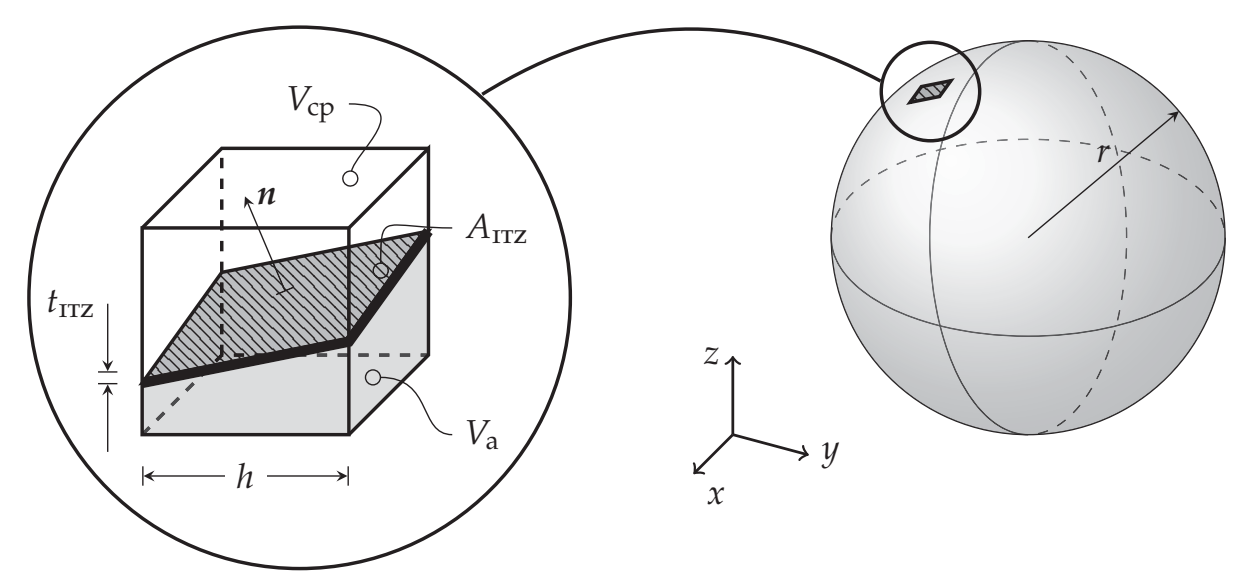

Figure 2: Interface voxel (left) located at the surface of a spherical aggregate (right). Element size is denoted $h$.

where $V_{\bullet}$ are volumes $\left[\mathrm{cm}^{3}\right], n$ and $A_{\text {ITZ }}$ is the normal and area $\left[\mathrm{cm}^{2}\right]$, respectively, of the ITZ as shown in Figure 2. Generally, we have that $D_{\mathrm{a}} \ll D_{\text {cp }} \ll D_{\text {IтZ }}$. The unknown parameters in Eq. (6) are $t$ and the diffusivity of ITZ, $D_{\text {ITZ }}$. Computationally, it is the product of these two, $D_{\text {Iтz }} \times t\left[\mathrm{~cm}^{3} \mathrm{~s}^{-1}\right]$, that become the model parameter and we introduce the auxiliary notation

$$
\begin{aligned}
& \hat{D}_{\mathrm{ITZ}} \stackrel{\text { def }}{=} D_{\mathrm{ITZ}} \times t, \\
& \hat{D}_{\mathrm{cp}} \stackrel{\text { def }}{=} D_{\mathrm{cp}} \times 1 \mathrm{~cm},
\end{aligned}
$$

to be used in Section 7 for the numerical examples.

To compute $A_{\mathrm{ITZ}}, V_{\mathrm{a}}$ and $V_{\mathrm{cp}}$, the intersection points between the aggregate surface and the line segments of the interface voxel need to be determined. The number of intersection points can range from 3 to 6 per voxel (Figure 2 shows 4 ) and are determined from the line-sphere intersection equation

$$
d=-(\boldsymbol{l} \cdot(\boldsymbol{o}-\boldsymbol{c})) \pm \sqrt{(\boldsymbol{l} \cdot(\boldsymbol{o}-\boldsymbol{c}))^{2}-(\boldsymbol{o}-\boldsymbol{c})^{2}+r^{2}}
$$

where $d$ is the intersection distance $[\mathrm{cm}]$ along the line's origin $\boldsymbol{o}, l$ is the direction of line (unit vector) and $r$ is the sphere's radius [cm]. For voxels close to an aggregate surface, $d$ is in practice computed for all of the voxel's 12 edge lines. There are three sets of solutions to $d$ :

$$
d \text { has } \begin{cases}2 \text { complex solutions } & \rightarrow \text { no i.p. }{ }^{1} \text { exits, } \\ 1 \text { real solution } & \rightarrow 1 \text { i.p. exits, } \\ 2 \text { real solutions } & \rightarrow 2 \text { i.p. exit. }\end{cases}
$$


$V_{\mathrm{a}}$ and $V_{\mathrm{cp}}$ in Figure 2 are convex hulls, see Berg et al. [4], spanned by these intersection points and the voxel's corner nodes, and are computed by utilizing this geometrical property. The interface area $A_{\mathrm{ITZ}}$, in turn, is determined by computing the enclosing areas of $V_{\mathrm{a}}$ and $V_{\mathrm{cp}}$ and utilizing the known total area of the voxel $\left(=6 h^{2}\right)$. In this fashion, the smooth aggregates' surfaces are represented as a discrete set of facets.

An alternative to use Eq. (6) would be to split the interface voxels in two parts by introducing new degrees of freedom (DOF) at the intersection points between the aggregate surface and line segments. In this way, $V_{\mathrm{a}}$ and $V_{\mathrm{cp}}$ could be subdivided into separate finite elements and $A_{\text {ITZ }}$ could be included as a separate 2D surface element. Such method has been proposed by Sohn et al. [26] in the context of elasticity using a so-called "carving technique". However, for the current problem of diffusion Eq. (6) suffices, as the numerical results in Section 7 show.

\subsection{Volumetric material description}

The aforementioned representation of the ITZ and the subdivision of interface voxels in terms of $V_{\mathrm{a}}$ and $V_{\mathrm{cp}}$ enables for an accurate volumetric representation of the cement paste and aggregate particles. Figure 3 shows numerically computed volume fraction of cement paste and aggregate surface area as a function of number of elements (NEL). These results correspond to an SVE with $L_{\square}=2 \mathrm{~cm}$ containing one single aggregate particle with a radius of $0.6 \mathrm{~cm}$, located at the center of the SVE.
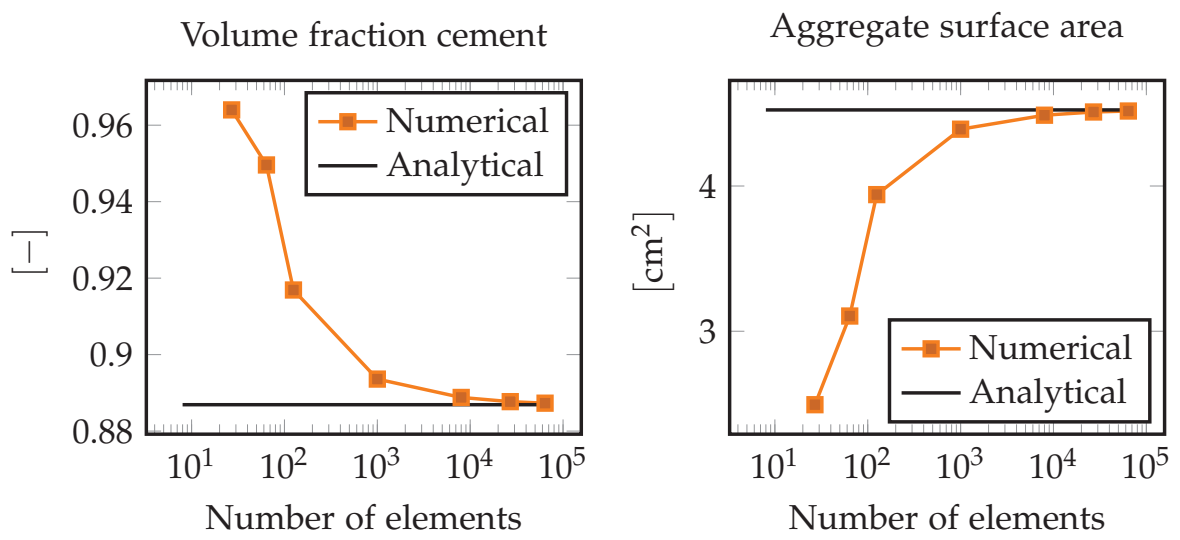

Figure 3: Convergence of volume fraction cement and aggregate surface area for an SVE.

Figure 3 shows that for a sufficiently dense mesh, the computed volume fraction of cement and the corresponding surface area of aggregates is in agreement with their analytical counterparts.

\footnotetext{
${ }^{1}$ i.p. $=$ intersection point.
} 


\section{First order homogenization}

Similar to Eq. (4), we wish to establish a macroscopic constitutive relation on the form

$$
\bar{J}=-\overline{\mathbf{D}} \cdot \bar{\nabla} \bar{\phi},
$$

where $\overline{-}$ denotes a macroscopic quantity, $\bar{J}$ is the macroscale flux, $\bar{D}$ is the macroscale diffusivity tensor and $\bar{\nabla} \bar{\phi}$ is the macroscale gradient of some macroscale potential $\bar{\phi}$. In particular, we want to determine $\overline{\boldsymbol{D}}^{\prime}$ 's dependence on aggregate content, diffusivity of ITZ and the number of spatial dimensions that the SVE comprise.

The homogenization procedure stems from the fully resolved, stationary, boundary value problem

$$
\nabla \cdot J=0 \quad \forall x \in \Omega,
$$

for which all material heterogeneities are embedded in $\Omega \subset \mathbb{R}^{3}$. Here, $\nabla$ is the nabla operator and $J(x)$ is the flux of some generic physical quantity. The corresponding weak form reads

$$
\int_{\Omega} \nabla \delta \phi \cdot J=\int_{\Gamma_{\mathrm{N}}} \delta \phi J \mathrm{~d} \Gamma
$$

for suitable test function $\delta \phi$ that is sufficiently regular.

Homogenization implies that the integrands of the volume integrals in Eq. (13) are replaced by space-averages on SVEs, i.e.

$$
\int_{\Omega} \nabla \delta \phi \cdot J \mathrm{~d} \Omega \longrightarrow \int_{\Omega}\langle\nabla \delta \phi \cdot J\rangle_{\square} \mathrm{d} \Omega,
$$

where $\langle\bullet\rangle_{\square}$ denotes the volume average

$$
\langle\bullet\rangle_{\square} \stackrel{\text { def }}{=} \frac{1}{\left|\Omega_{\square}\right|} \int_{\Omega_{\square}} \bullet \mathrm{d} \Omega_{\square},
$$

on the SVE that occupies the domain $\Omega \square$ centered at the macroscale position $\bar{x} \in \Omega$; hence, $\langle x-\bar{x}\rangle_{\square}=0$.

We assume first order homogenization defined by the split of a scalar field $\phi$ within $\Omega_{\square}$ into the macroscale and fluctuation parts ${ }^{2}$ as follows:

$$
\phi(x ; \bar{x})=\phi^{\mathrm{M}}(x ; \bar{x})+\phi^{\mathrm{s}}(x) .
$$

The macroscale part varies linearly as

$$
\phi^{\mathrm{M}}(x ; \bar{x})=\bar{\phi}(\bar{x})+\bar{g}_{\phi}(\bar{x}) \cdot[x-\bar{x}] \quad \forall x \in \Omega_{\square},
$$

with $\bar{g} \stackrel{\text { def }}{=} \bar{\nabla} \bar{\phi}$. Hence, we obtain $\nabla \phi^{\mathrm{M}}(\bar{x} ; \bar{x})=\bar{g}(\bar{x})$ constant within $\Omega_{\square}$. We then obtain for any macroscale point $\bar{x} \in \Omega$ the identity

\footnotetext{
${ }^{2}$ Superscripts $M$ denotes macroscale, whereas s denotes subscale.
} 


$$
\langle\nabla \delta \phi \cdot J\rangle_{\square}=\delta \overline{\boldsymbol{g}} \cdot \overline{\boldsymbol{J}} \quad \text { with } \quad \overline{\boldsymbol{J}}=\langle\boldsymbol{J}\rangle_{\square} .
$$

In order to compute the homogenized flux quantity $\bar{J}$, it is necessary to compute the fluctuation field $\phi^{\mathrm{s}}$ as well as the spatial gradient on the subscale: $g^{\mathrm{s}} \stackrel{\text { def }}{=} \nabla \phi^{\mathrm{s}}$. This is done on each SVE with given prolongation conditions. Here, we choose Dirichlet boundary conditions, i. e. $\phi^{\mathrm{s}}=0$ on $\Gamma_{\square}$, whereby the pertinent SVE problem becomes: For given values of the macroscale variables $\bar{\phi}$ and $\bar{g}$, compute the subscale field $\phi^{\mathrm{s}}$ that satisfy the system

$$
\left\langle\nabla\left(\delta \phi^{\mathrm{s}}\right) \cdot \boldsymbol{J}\right\rangle_{\square}=0,
$$

for all possible $\delta \phi^{\mathrm{s}}$ that are sufficiently regular and which vanish on $\Gamma_{\square}$. We note that the macroscale prolongations $\phi^{\mathrm{M}}$ are completely defined (varies linearly) on $\Omega_{\square}$.

\section{Linearized macroscopic problem}

In the general situation it is necessary to solve the macroscale problem Eq. (13) and the corresponding SVE problem in each macroscale quadrature point in a "nested" fashion, known as an $\mathrm{FE}^{2}$ procedure. The nested problem is conveniently solved via Newton iterations on the macroscale. It is then necessary to find the macroscale tangent relations which represent linearizations of the (implicit) functions $\overline{\boldsymbol{J}}\{\bar{\phi} ; \overline{\boldsymbol{g}}\}$ :

$$
\mathrm{d} \bar{J}=-\bar{D} \cdot \mathrm{d} \bar{g}-\bar{Y} \mathrm{~d} \bar{\phi},
$$

which requires the solution of the appropriate "sensitivity problem" (or tangent problem) associated with each SVE.

However, in this paper we shall not consider solution obtained through $\mathrm{FE}^{2}$ computations but rather make simplifications in order to derive upscaled macroscale constitutive relations. Such relations are obtained upon assuming linearization for vanishing macroscale spatial gradients, i.e. at the state $\bar{g}=\mathbf{0}$. The linearized expressions are then taken as valid for "small" changes of the macroscale spatial gradients. For this situation we obtain $\phi=\phi^{\mathrm{M}}+\phi^{\mathrm{S}}=\bar{\phi}+\phi^{\mathrm{S}}$ and the constitutive relation in Eq. (4) becomes

$$
J=-D \cdot \nabla \phi^{\mathrm{s}},
$$

with ts variation

$$
\mathrm{d} \boldsymbol{J}=-\boldsymbol{D} \cdot \boldsymbol{\nabla}\left(\mathrm{d} \phi^{\mathrm{s}}\right) .
$$

The sensitivity problem corresponding to variations of the macroscale fields $\bar{\phi}$ and $\bar{g}$ can be expressed as

$$
\left\langle\nabla\left(\delta \phi^{\mathrm{s}}\right) \cdot \mathrm{d} \boldsymbol{J}\right\rangle_{\square}=0,
$$


for all possible $\delta \phi^{\mathrm{s}}$ that is sufficiently regular and which vanish on $\Gamma_{\square}$. Upon inserting Eq. (22) into Eq. (23), we obtain the tangent relation

$$
\left\langle\nabla\left(\delta \phi^{\mathrm{s}}\right) \cdot \boldsymbol{D} \cdot \nabla(\mathrm{d} \phi)\right\rangle_{\square}=0 .
$$

\subsection{Sensitivity fields for SVE problem}

To determine the relations in Eq. (20) explicitly, we shall be interested in computing unit fluctuation fields, i.e. sensitivity fields, corresponding to unit values of $\mathrm{d} \bar{\phi}$ and $\mathrm{d} \bar{g}$. Hence, we need to compute the differentials $\mathrm{d} \phi=\mathrm{d} \phi^{\mathrm{M}}+\mathrm{d} \phi^{\mathrm{s}}$ in terms of $\mathrm{d} \bar{\phi}$ and $\mathrm{d} \overline{\boldsymbol{g}}_{\phi}$. Firstly, we conclude that

$$
\mathrm{d} \phi^{M}(\boldsymbol{x})=\mathrm{d} \bar{\phi}+\mathrm{d} \overline{\boldsymbol{g}} \cdot[\boldsymbol{x}-\overline{\boldsymbol{x}}]=\mathrm{d} \bar{\phi}+\sum_{i=1}^{N D I M} \hat{\phi}^{M(i)}(\boldsymbol{x}) \mathrm{d} \bar{g}_{i}
$$

where NDIM is the number of spatial dimensions. The unit fields, $\hat{\phi}^{M(i)}(\boldsymbol{x})$, is given as

$$
\hat{\phi}^{M(i)}(x)=e_{i} \cdot[x-\bar{x}]=x_{i}-\bar{x}_{i} \quad \Rightarrow \quad \nabla \hat{\phi}^{M(i)}(x)=e_{i} .
$$

Next, we introduce the following ansatz for $\mathrm{d} \phi^{s}$ :

$$
\mathrm{d} \phi^{\mathrm{s}}(\boldsymbol{x})=\hat{\phi}_{\bar{\phi}}^{\mathrm{s}}(\boldsymbol{x}) \mathrm{d} \bar{\phi}+\sum_{i=1}^{N D I M} \hat{\phi}_{\overline{\boldsymbol{g}}}^{\mathrm{s}(i)}(\boldsymbol{x}) \mathrm{d} \bar{g}_{i}
$$

and we thus obtain the expressions for the total differential, $\mathrm{d} \phi$, in terms of the sensitivities as

$$
\begin{aligned}
\mathrm{d} \phi(x)= & {\left[1+\hat{\phi}_{\bar{\phi}}^{\mathrm{s}}(\boldsymbol{x})\right] \mathrm{d} \bar{\phi} } \\
& +\sum_{i=1}^{N D I M}\left(\left[\hat{\phi}^{\mathrm{M}(i)}(\boldsymbol{x})+\hat{\phi}_{\overline{\boldsymbol{g}}}^{\mathrm{s}(i)}(\boldsymbol{x})\right] \mathrm{d} \bar{g}_{i}\right) .
\end{aligned}
$$

We show that the sensitivity $\hat{\phi}_{\bar{\phi}}^{s}$ vanish by consider the case $\mathrm{d} \bar{\phi} \neq \mathbf{0}$ while all other macroscale differentials are zero. Upon inserting the expressions in Eq. (28) into Eq. (24), we identify the system

$$
\left\langle\nabla\left(\delta \phi^{\mathrm{s}}\right) \cdot \boldsymbol{D} \cdot \nabla \hat{\phi}_{\bar{\phi}}^{\mathrm{s}}\right\rangle_{\square}=0,
$$

Setting $\delta \phi^{\mathrm{s}}=\phi_{\bar{\phi}}^{\mathrm{s}}$ in Eq. (29), we obtain 


$$
\left\langle\nabla\left(\hat{\phi}_{\bar{\phi}}^{\mathrm{s}}\right) \cdot \boldsymbol{D} \cdot \nabla \hat{\phi}_{\bar{\phi}}^{\mathrm{s}}\right\rangle_{\square}=0
$$

which only holds for $\hat{\phi}_{\bar{\phi}}^{\mathrm{s}}=0$ since $\boldsymbol{D} \neq \mathbf{0}$. In summary, we have

$$
\mathrm{d} \phi(x)=\mathrm{d} \bar{\phi}+\sum_{i=1}^{N D I M}\left[\hat{\phi}^{\mathrm{M}(i)}(\boldsymbol{x})+\hat{\phi}^{\mathrm{s}(i)}(\boldsymbol{x})\right] \mathrm{d} \bar{g}_{i}
$$

where the simplified notation $\hat{\phi}^{\mathrm{s}(i)} \stackrel{\text { def }}{=} \hat{\phi}_{\bar{g}}^{\mathrm{s}(i)}$ was introduced. The remaining equation become

$$
\left\langle\nabla\left(\delta \phi^{\mathrm{s}}\right) \cdot \boldsymbol{D} \cdot \boldsymbol{\nabla} \hat{\phi}^{\mathrm{s}(i)}\right\rangle_{\square}=-\left\langle\nabla\left(\delta \phi^{\mathrm{s}}\right) \cdot \boldsymbol{D} \cdot \boldsymbol{e}_{i}\right\rangle_{\square}
$$

from which $\hat{\phi}^{\mathrm{s}(i)}$ can be computed.

\subsection{Homogenized linearized diffusivity tensors}

We can now evaluate the macroscale diffusivity tensors (defined via linearization) occurring in Eq. (20) by first noting the identity

$$
\mathrm{d} \bar{J}_{i}=\left\langle\mathrm{d} \boldsymbol{J} \cdot \boldsymbol{e}_{i}\right\rangle_{\square}
$$

and combining with Eq. (22) and Eq. (31) to obtain (i) $\bar{Y}=\mathbf{0}$ and (ii) the non-zero tangent tensors in terms of their components

$$
(\overline{\boldsymbol{D}})_{i j}=\langle\boldsymbol{D}\rangle_{\square}+\sum_{i=1}^{3}\left\langle\nabla\left(\hat{\phi}^{s(i)}\right) \cdot \boldsymbol{D}\right\rangle_{\square} \otimes \boldsymbol{e}_{i}
$$

and for the numerical examples in the following section, we introduce the auxiliary definition of the effective macroscopic diffusivity as

$$
D_{\text {eff }} \stackrel{\text { def }}{=}(\overline{\boldsymbol{D}})_{11} \text {. }
$$




\section{Numerical examples}

\subsection{Preliminaries}

Our first objective is to determine the mapping

$$
L_{\square} \mapsto D_{\text {eff }}\left(L_{\square}\right)
$$

and to study how this mapping differs between 3D and 2D SVEs. This is done by solving Eq. (19) given a macroscale gradient $\bar{g}=\left[\begin{array}{lll}-1 & 0 & 0\end{array}\right]^{\mathrm{T}}$ for SVEs of varying $L_{\square}$. The aggregates were assigned a diffusivity of $D_{\mathrm{a}}=0 \mathrm{~cm}^{2} \mathrm{~s}^{-1}$. Only linear problems are treated herein and all numerical results are normalized with respect to $D_{\mathrm{cp}}$. Hence, the numerical value of $D_{\mathrm{cp}}$ chosen will bear no influence on the reported results in this section. For reference, a realistic value of $D_{\mathrm{cp}}$ is in the range of $2 \times 10^{-8} \mathrm{~cm} \mathrm{~s}^{-1}$ to $20 \times 10^{-8} \mathrm{~cm} \mathrm{~s}^{-1}$.

A set of SVEs was generated with an aggregate volume fraction of $42 \%$ with a sieve curve similar to the one used in Wriggers and Moftah [29], as shown in Figure 4. The SVEs were of different sizes, $L_{\square}=2 \mathrm{~cm}, 4 \mathrm{~cm}, 6 \mathrm{~cm}, 8 \mathrm{~cm}, 10 \mathrm{~cm}$ and $12 \mathrm{~cm}$, and $40 \mathrm{SVE}$ realizations were made for each value of $L_{\square}$. The mesh size was set to $h=0.029 \mathrm{~cm}$, based on convergence studies as shown in Figure 3.

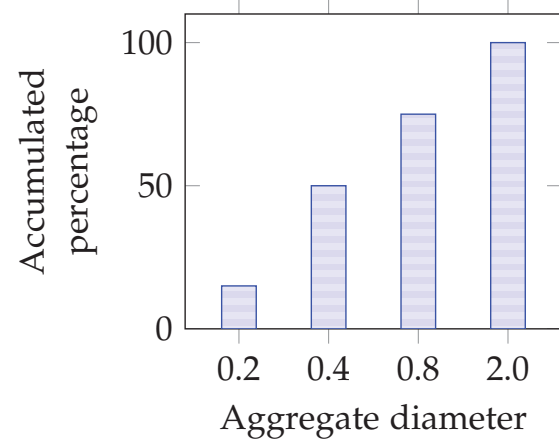

Figure 4: Sieve curve for all SVEs used in the numerical examples.

\subsection{D SVEs}

The 2D SVEs were generated by cutting a 3D SVE into a number of 2D slices, see Figure 5. The same boundary conditions were applied to the 3D and 2D SVEs and the mean values of all slices were then compared to the single result obtained for the corresponding 3D SVE. 


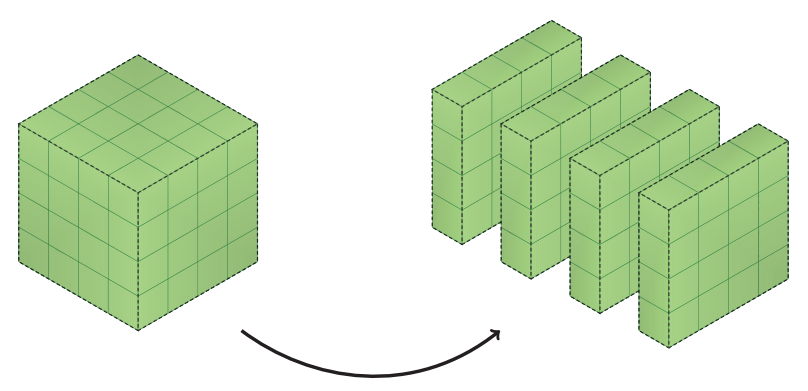

Figure 5: 3D SVE cut into 2D slices.

\subsection{D/2D comparison and influence of ITZ}

The numerical results in Figure 6 show effective diffusivities, $D_{\text {eff }}$, as a function of $L_{\square}$ for 3D and 2D SVEs and varying ITZ diffusivities. $D_{\text {eff }}^{2 D}$ is lower than $D_{\text {eff }}^{3 \mathrm{D}}$ for all values of $L_{\square}$ and $\hat{D}_{\text {ITZ}}$, which is expected since the out of plane solution for the 2D SVEs is locked. Hence, the diffusing substance only has two directions (over or under) to bypass aggregates in 2D SVEs but for 3D SVEs the third spatial direction (around) is also enabled which decrease resistance and, thus, increases diffusivity.

It is further noticed in Figure 6 that $D_{\text {eff }}$ is more or less invariant to $L_{\square}$ for all values of $\hat{D}_{\text {ITz }}$. The only exception is $D_{\text {eff }}^{2 D}$ for $\hat{D}_{\text {ITz }}=0$ where a converged value is reached only at $L_{\square}=10 \mathrm{~cm}$.

Figure 43 show that $D_{\text {eff }}^{3 \mathrm{D}}$ is about $17 \%$ greater than $D_{\text {eff }}^{2 \mathrm{D}}$ when ITZ is included in the SVEs. The corresponding difference for SVEs without ITZ is almost $40 \%$ for $L_{\square}=10 \mathrm{~cm}$.

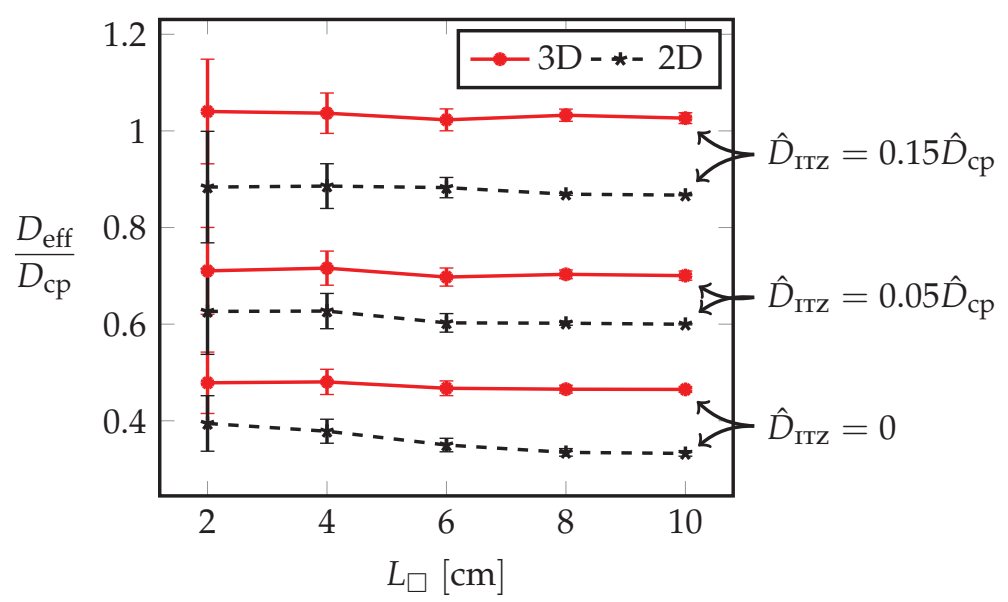

Figure 6: Effective diffusivities as functions of SVE size and ITZ diffusivities. Error bars show mean values, $\mu, \pm$ one standard deviation, $\sigma$. 


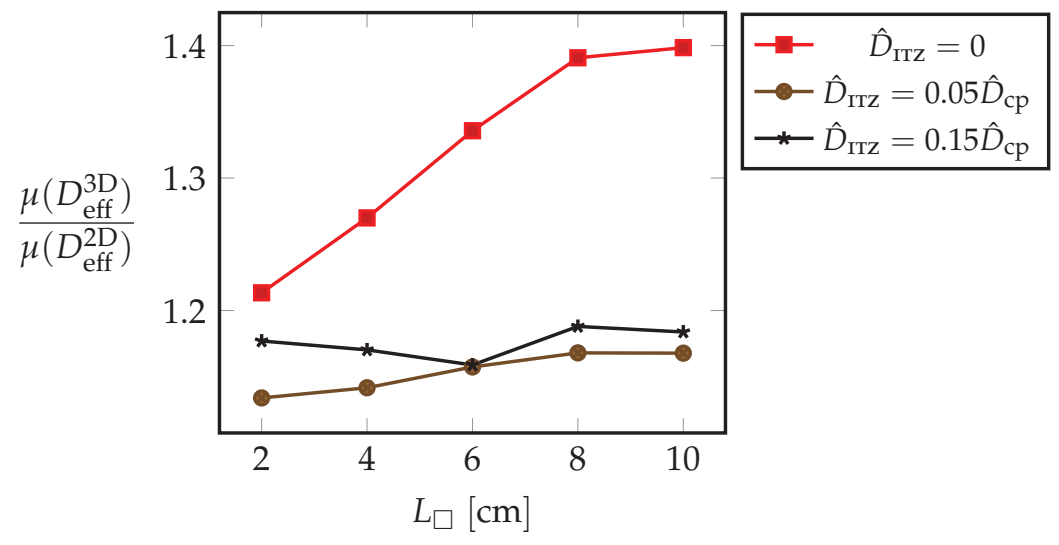

Figure 7: Difference in effective diffusivities for 3D and 2D SVEs. Quotient between mean values, $\mu$, are plotted.

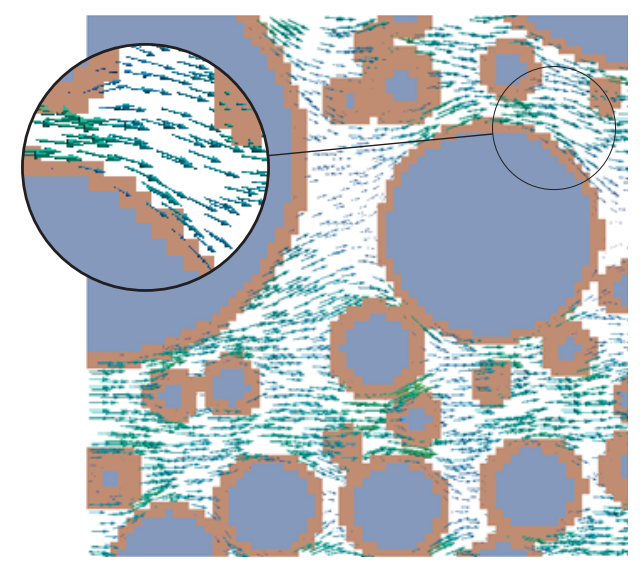

(a) $\hat{D}_{\text {ITZ }}=0$ (no ITZ).

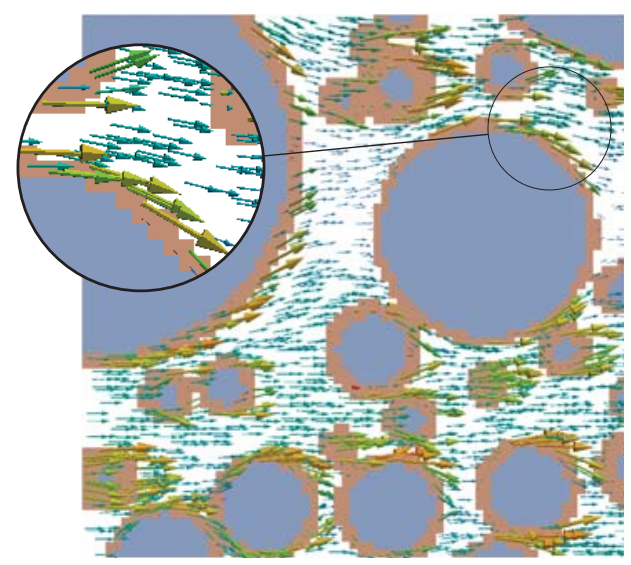

(b) $\hat{D}_{\text {ITZ }}=0.15 \hat{D}_{\text {cp }}$ (with ITZ).

Figure 8: The effect of ITZ in the mesoscale structure by visual comparison. Flux arrows are larger in magnitude at the aggregate surfaces when ITZ is included the SVEs.

The results in Figure 6 show that it is possible to increase the effective diffusivity by utilizing the ITZ implementation as a model parameter. The results also show the robustness of the implementation as the mean values in practice become independent of the SVE size, $L_{\square}$. It is only the spread in results that varies, which also decreases with increasing $L_{\square}$, as expected.

The value $\hat{D}_{\text {ITz }}=0.15 \hat{D}_{\text {cp }}$ has been manually calibrated such that $D_{\text {eff }}$ gets close to a value corresponding to the diffusivity of the pure cement paste, $D_{\mathrm{cp}}$. 
The influence of ITZ on the diffusion flux within the SVE is shown in Figure 8. Two observations can be made: the flux field is more horizontally oriented, and the flux arrows are larger in magnitude close to the aggregate surface when ITZ is included. This implies that the diffusing substance is attracted to the aggregates when ITZ is included, and repulsed when ITZ is omitted.

The outcome of this numerical example is that the over-all effect of the aggregates can be controlled and modified. This is achieved by the ITZ implementation. In this way, the parameter $\hat{D}_{\text {IтZ }}$ becomes a useful tool for calibrating the model with experimental data. Additionally, the mean value of $D_{\text {eff }}^{3 D}$ is more or less invariant to the SVE size, only the scatter in results decrease with increasing $L_{\square}$. This is beneficial since smaller SVEs can be used, which reduces computational cost dramatically since an SVE's DOFs grows by $\mathcal{O}\left(n^{3}\right)$ with increasing $L_{\square}$ and fixed element size $h$.

\subsection{Bounds on effective diffusivity}

The numerical results obtained through computational homogenization $(\mathrm{CH})$ are compared to analytical and numerical bounds, which are described in the following. These are all upper bounds since the corresponding lowers ones, i.e. lower Hashin-Shtrikman bound $\left(\mathrm{HS}^{-}\right.$) and Reuss, all become zero due to the choice $D_{\mathrm{a}}=0 \mathrm{~cm}^{2} \mathrm{~s}^{-1}$.

Voigt bound The sieve curve dependent Voigt bound is expressed as the arithmetic mean

$$
D_{\text {eff }}^{\text {Vigt }}=D_{\mathrm{cp}}-n_{\mathrm{a}} \sum_{i=1}^{N}\left(\frac{4 f_{i}}{r_{i}} \hat{D}_{\mathrm{ITZ}}-f_{i} D_{\mathrm{cp}}\right)
$$

whose derivation can be found in Appendix A.

Hashin-Shtrikman bound The upper Hashin-Shtrikman bound $\left(\mathrm{HS}^{+}\right)$[12] is determined from

$$
D_{\mathrm{eff}}^{\mathrm{Hs}+}=D_{\mathrm{cp}}+\frac{n_{\mathrm{a}}}{\frac{1}{D_{\mathrm{a}}-D_{\mathrm{cp}}}+\frac{1-n_{\mathrm{a}}}{3 D_{\mathrm{cp}}}},
$$

which for the choice $D_{\mathrm{a}}=0 \mathrm{~cm}^{2} \mathrm{~s}^{-1}$ and normalization with respect to $D_{\mathrm{cp}}$ simplifies to

$$
D_{\text {eff }}^{\mathrm{HS}^{+}}=\frac{2-2 n_{\mathrm{a}}}{2+n_{\mathrm{a}}} \mathrm{cm}^{2} \mathrm{~s}^{-1},
$$

$\mathrm{HS}^{+}$is included since it is reported in the literature to be tighter a bound than Voigt for materials with spherical inclusion, see e.g. Stora et al. [27], Gross and Seeling [9] and Mehta and Monteiro [18]. However, $\mathrm{HS}^{+}$is only valid asymptotically for infinitely large SVEs, see Zohdi and Wriggers [32]. 
Taylor with homogenized ITZ surface The scalar field $\phi$ is assumed to vary linearly within the SVE, i.e.

$$
\begin{aligned}
\phi(x ; \bar{x}) & =\phi^{\mathrm{M}}(x ; \bar{x})+\phi^{\mathrm{s}}(x)^{-0} \\
& =\bar{\phi}(\bar{x})+\bar{g}_{\phi}(\bar{x}) \cdot[x-\bar{x}] \quad \forall x \in \Omega_{\square},
\end{aligned}
$$

and no SVE problem is solved to determine $D_{\text {eff }}$. The Taylor assumption accounts for the SVE's topology, which is not the case for the $\mathrm{HS}^{+}$and Voigt bounds.

\subsection{Influence of aggregate content}

A set of SVEs were generated for a fixed size $L_{\square}=6 \mathrm{~cm}$, with a varying aggregate content, $n_{\mathrm{a}}$. 20 SVE realizations were made for each value of $n_{\mathrm{a}}$.

The choice $L_{\square}=6 \mathrm{~cm}$ was made based on the results in Figure 6 as the scatter in results was sufficiently small for this SVE size. The same sieve curve as in Figure 4 was used to generate the SVEs. The total aggregates' total surface area for these SVEs scale linearly with $n_{\mathrm{a}}$ as can be seen in Figure 9. A snapshot of the stationary solution in Figure 10 shows how the streamlines in the cement paste curve around the aggregates.

Figure 11 shows $D_{\text {eff }}\left(n_{\mathrm{a}}\right)$ for varying values of $\hat{D}_{\text {ITz }}$, along with the different types of bounds. The numerical results for $\hat{D}_{\text {ITz }}=0$ coincide with $\mathrm{HS}^{+}$and are below the Voigt bound. The Voigt bound, in turn, coincide the Taylor assumption for $\hat{D}_{\text {ITz }}=0$. This validates the model for the case of a two-phase mesoscale structure of cement and aggregates (no ITZ) as the Voigt bound is a true upper bound.

Total $A_{\text {ITz }}$ per SVE.

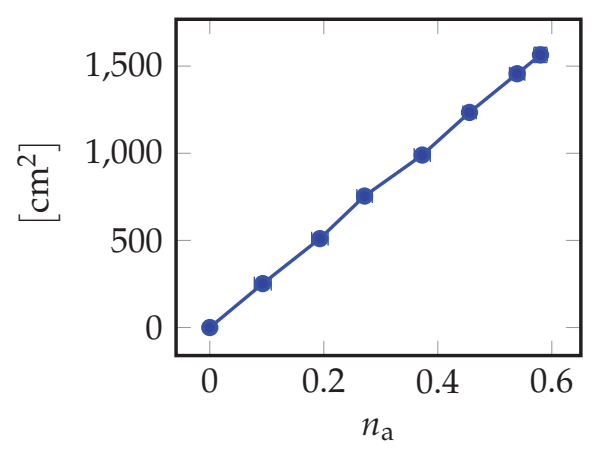

Figure 9: Total aggregate surface area as function of $n_{\mathrm{a}}$ for the set of SVEs with $L_{\square}=6 \mathrm{~cm}$. 


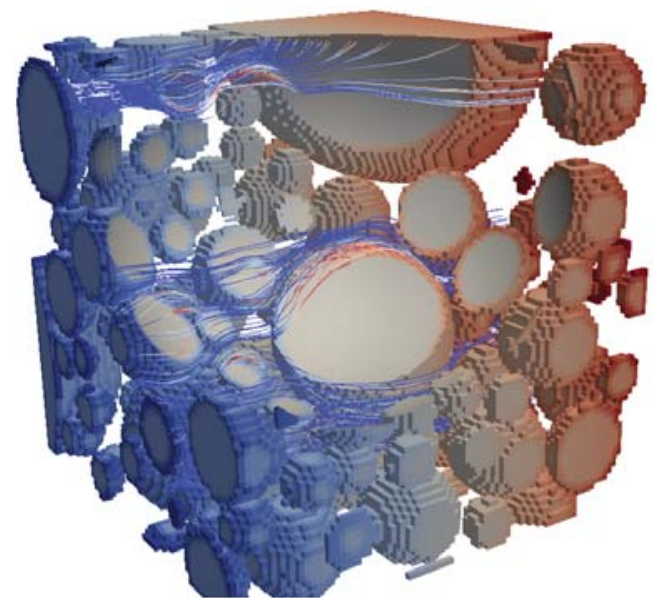

Figure 10: Stationary solution showing diffusion streamlines. Colour gradient represents stationary solution at the surface of the aggregates.

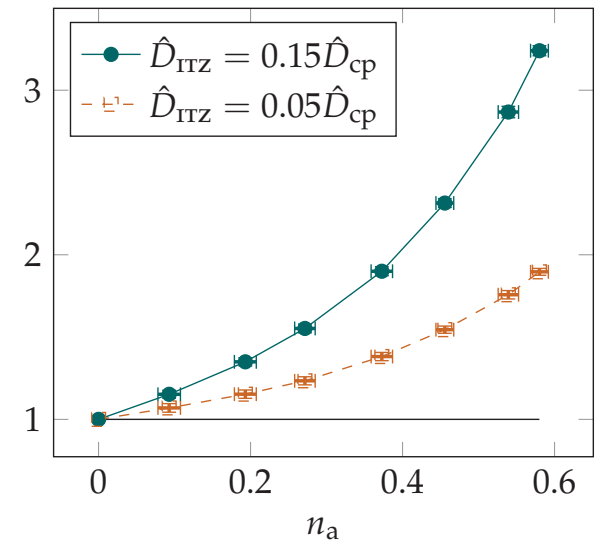

Figure 12: $\frac{D_{\text {eff }}}{D_{\mathrm{cp}}}$ normalized with $\hat{D}_{\mathrm{ITZ}}=0$.

However, when the ITZ is included in the model the effective diffusivity takes values above the $\mathrm{HS}^{+}$and Voigt bounds since the model now constitutes a three-phase material. Work by Nilsen and Monteiro [20] show that the elastic modulus of concrete, determined from experiments, lay outside the $\mathrm{HS}^{+}-\mathrm{HS}^{-}$bounds. The authors argues that this observation can be derived from the presence of ITZ and they suggest therefore concrete to be considered a three-phase material on the mesoscale. Corresponding experimental work for transport properties is currently not readily available in the literature but to consider concrete only a two-phase material on the mesoscale is most probably not adequate for diffusion phenomena either. 

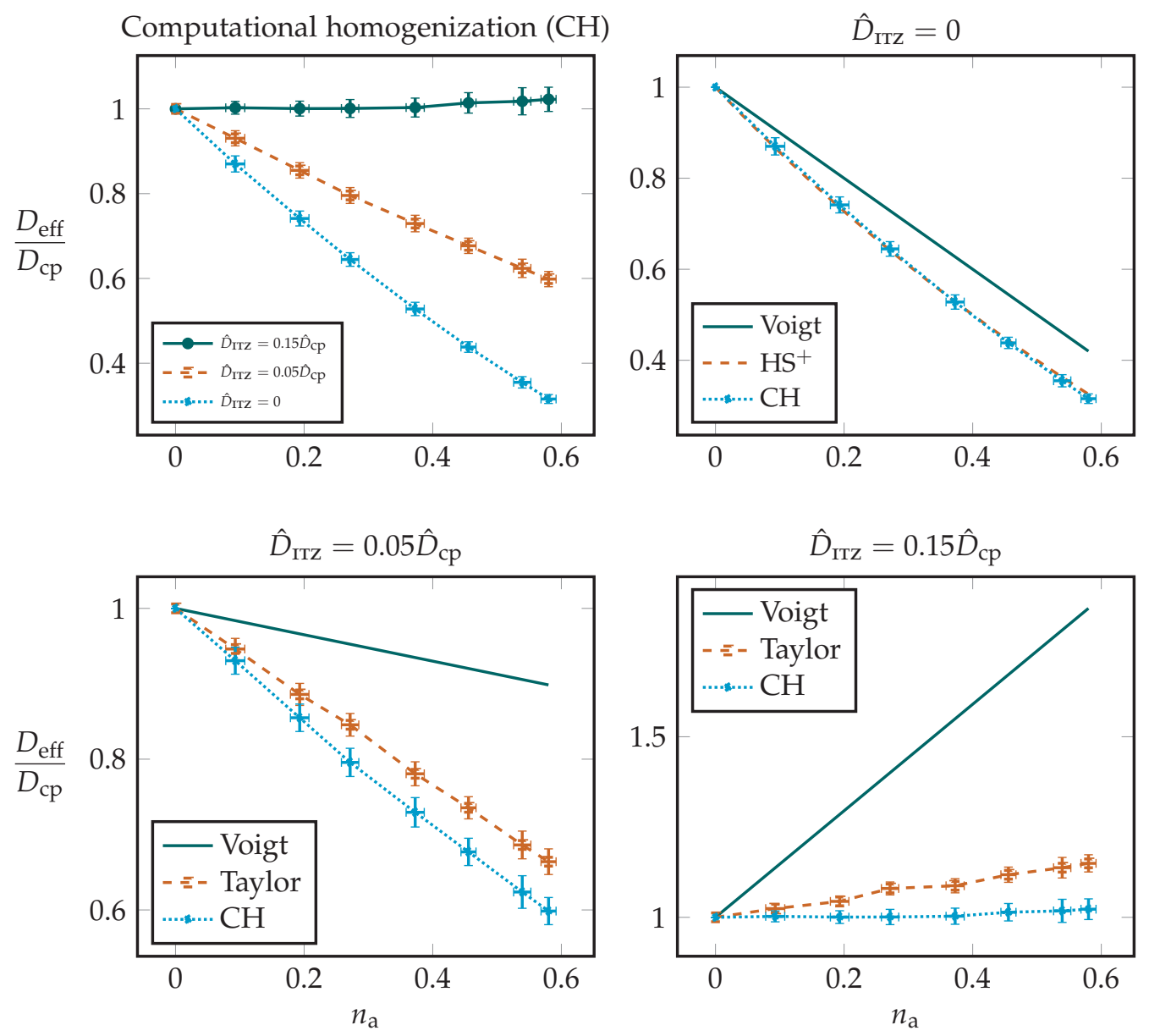

Figure 11: Effective diffusivity as a function of aggregate content, $n_{\mathrm{a}}$. Error bars show mean values \pm one standard deviation for both $D_{\text {eff }}$ and $n_{\mathrm{a}}$. 
As stated, $\hat{D}_{\text {ITZ }}$ specifies neither the diffusivity of the ITZ $D_{\text {ITZ, }}$, nor its thickness, $t$, but only the product of the two. A realistic value of $t$ is in the range of $20 \mu \mathrm{m}$ to $50 \mu \mathrm{m}$, c.f. Yang and Su [30]. The relation $\hat{D}_{\text {ITZ }}=0.05 \hat{D}_{\mathrm{cp}}$, assuming $t=40 \mu \mathrm{m}$, can thus be re-written as

$$
\begin{aligned}
D_{\text {ITZ }} \times 40 \times 10^{-6} \mathrm{~m} & =0.05 D_{\mathrm{cp}} \times 0.01 \mathrm{~m} \\
\Rightarrow \frac{D_{\text {ITZ }}}{D_{\mathrm{cp}}} & =12.5,
\end{aligned}
$$

and the corresponding ratio for $\hat{D}_{\mathrm{ITZ}}=0.15 \hat{D}_{\mathrm{cp}}$ is

$$
\frac{D_{\mathrm{ITZ}}}{D_{\mathrm{cp}}}=37.5 .
$$

The ratio in Eq. (43) is directly dependent on the chosen sieve curve - since the total ITZ area in each SVE will vary with varying sieve curves - and the aggregate contents. By Eq. (43) the diffusivity of the ITZ could be determined by calibrating the relation between $\hat{D}_{\text {IтZ }}$ and $\hat{D}_{\text {cp }}$ with experimental data. Experimentally obtained ratios of Eq. (43) reported in the literature ranges from 2 to 12, c.f. Yang and Su [30], Ollivier et al. [21]; hence, the numerical ratio in Eq. (43) is reasonable in magnitude.

Figure 12 shows that $D_{\text {eff }}$ increases non-linearly with increasing $n_{\mathrm{a}}$ even thou the total aggregate surface area increases linearly with increasing $n_{\mathrm{a}}$ as see in Figure 9 .

Influence of topology As can be observed in Figure 11, the Voigt bound deviates from $\mathrm{CH}$ with increasing $n_{\mathrm{a}}$ and increasing $\hat{D}_{\text {IтZ }}$. However, the Taylor assumption is in rather good agreement with $\mathrm{CH}$ for $\hat{D}_{\text {Iтz }}>0$. This implies that information on material topology greatly influences the effective diffusivity since the Voigt bound only considers volume fractions of each material phase. If the topology is known, then applying the Taylor assumption yields a good approximation of the effective diffusivity and might even be sufficient. However, if concrete can be considered a two phase material then $\mathrm{HS}^{+}$is sufficient to use over $\mathrm{CH}$.

\subsection{Computational time}

The computational time per SVE, $t_{\mathrm{SVE}}[\mathrm{s}]$, to determine $D_{\text {eff }}$ is

$$
t_{\mathrm{SVE}} \approx 0.0003 \times \mathrm{NEL}
$$

running MatLAB ver. 7.14 (2012a) on AMD CPUs with a clock speed of 3 GHz. MatLAB's implementation of the minimal residual method is used to iteratively solve the system of equations. The maximum number of elements in this work was $260^{3}=17,576,000$; hence, $t_{\mathrm{CPU}}^{\max } \approx 80 \mathrm{~min}$. However, the implementation is parallelized, i.e. multiple SVE problems can be solved concurrently, and the total computational time is therefore mainly governed by the number of CPU-cores available. 


\section{Conclusions and outlook}

A 3D model of heterogeneous mesoscale concrete was presented and employed for computational homogenization $(\mathrm{CH})$ in the context of mass diffusion. The algorithm on which the model is based generates a random structure of aggregates embedded in cement paste within a Statistical Volume Element (SVE). Any sieve curve is possible as input for the algorithm and any (realistic) aggregate content is possible to obtain.

For the numerical examples presented, the effective diffusivity for 3D SVEs is about $17 \%$ greater than for 2D SVEs. The corresponding difference when the Interfacial Transition Zone (ITZ) is omitted from the SVEs is $40 \%$. This significant difference between 3D and 2D SVE shows the importance of accounting for all three spatial dimensions when calibrating a diffusion model with experimental data.

Additionally, the numerical results coincide with the upper Hashin-Shtrikman bound $\left(\mathrm{HS}^{+}\right)$in the case of a two-phase mesoscale model of cement paste and aggregates. For three phase concrete, the Taylor assumption yields good estimate compared with $\mathrm{CH}$ of the effective diffusivity in the case of linear material properties on the mesoscale.

The numerical results further show that the ITZ implementation can be used to increase the effective diffusivity by an arbitrary scale factor in an easy and efficient way. This enables the model to be used for calibrating the ITZ's diffusivity with experimental data, which is to be done by carrying out transient analyses.

\section{Acknowledgments}

This research was financially supported by The Swedish Research Council, which is gratefully acknowledged. The computations were performed on resources at Chalmers Centre for Computational Science and Engineering $\left(\mathrm{C}^{3} \mathrm{SE}\right)$ provided by the Swedish National Infrastructure for Computing (SNIC). 


\section{A Voigt bound}

$V_{\text {ITZ }}$ is given by

$$
\begin{aligned}
V_{\text {ITZ }} & =\frac{4}{3} \pi(r+t)^{3}-\frac{4}{3} \pi r^{3} \\
& =\{t \ll r\} \approx \frac{16 \pi}{3} r^{2} t,
\end{aligned}
$$

where $t$ and $r$ are the thickness of the ITZ and the aggregate radius, respectively, which yields

$$
\frac{V_{\mathrm{ITZ}}}{V_{\mathrm{a}}}=\frac{4 t}{r} \Rightarrow n_{\mathrm{ITZ}}=\frac{4 t}{r} n_{\mathrm{a}}=n_{\mathrm{a}} \sum_{i=1}^{N} \frac{4 t}{r_{i}} f_{i} .
$$

The Voigt bound then takes the form

$$
\begin{aligned}
D_{\text {eff }}^{\text {Voigt }}\left(n_{\mathrm{a}}\right) & =n_{\mathrm{a}} D_{\mathrm{a}}+n_{\mathrm{cp}} D_{\mathrm{cp}}+n_{\mathrm{ITZ}} D_{\mathrm{ITZ}} \\
& =n_{\mathrm{a}} D_{\mathrm{a}}+n_{\mathrm{cp}} D_{\mathrm{cp}}+n_{\mathrm{a}} \sum_{i=1}^{N} \frac{4 f_{i}}{r_{i}} \hat{D}_{\mathrm{ITZ}} \\
& =\left\{n_{\mathrm{a}}+n_{\mathrm{cp}} \approx 1\right\} \\
& =n_{\mathrm{a}} D_{\mathrm{a}}+\left(1-n_{\mathrm{a}}\right) D_{\mathrm{cp}}+n_{\mathrm{a}} \sum_{i=1}^{N} \frac{4 f_{i}}{r_{i}} \hat{D}_{\mathrm{ITZ}} \\
& =n_{\mathrm{a}} D_{\mathrm{a}}+D_{\mathrm{cp}}+n_{\mathrm{a}} \sum_{i=1}^{N}\left(\frac{4 f_{i}}{r_{i}} \hat{D}_{\mathrm{ITZ}}-f_{i} D_{\mathrm{cp}}\right) \\
& =\left\{D_{\mathrm{a}}=0 \mathrm{~cm}^{2} \mathrm{~s}^{-1}\right\} \\
& =D_{\mathrm{cp}}+n_{\mathrm{a}} \sum_{i=1}^{N}\left(\frac{4 f_{i}}{r_{i}} \hat{D}_{\mathrm{ITZ}}-f_{i} D_{\mathrm{cp}}\right) .
\end{aligned}
$$

This bound is valid for $\hat{D}_{\text {ITz }}=0$ but not for high values of $n_{\mathrm{a}}$ while $\hat{D}_{\mathrm{ITZ}}>0$ since $D_{\text {eff }}^{\text {Voigt }}\left(n_{\mathrm{a}}=1, \hat{D}_{\text {ITz }}>0\right) \neq D_{\mathrm{a}}$.

\section{References}

[1] D. Asahina and J.E. Bolander. Voronoi-based discretizations for fracture analysis of particulate materials. Powder Technology, 213(1-3):92-99, November 2011. ISSN 00325910. doi: 10.1016/j.powtec.2011.07.010.

[2] Dale P. Bentz, Edward J. Garboczi, Kenneth A. Snyder. A hard core/soft shell microstructural model for studying percolation and transport in three-dimensional composite media. U.S. Dept. of Commerce, Technology Administration, National Institute of Standards and Technology, 1999. 
[3] D Bentz, D Quenard, H Kunzel, J Baruchel, F Peyrin, N Martys and E Garboczi. Microstructure and transport properties of porous building materials. II: Three-dimensional X-ray tomographic studies. Materials and Structures, 33(3):147-153, 2000. doi: 10.1007/ BF02479408.

[4] Mark de Berg, Otfried Cheong, Marc van Kreveld, and Mark Overmars. Computational Geometry: Algorithms and Applications. Springer-Verlag TELOS, Santa Clara, CA, USA, 3rd ed. edition, 2008. ISBN 3540779736, 9783540779735.

[5] A Caballero, C Lopez, and I Carol. 3D meso-structural analysis of concrete specimens under uniaxial tension. Comput. Methods Appl. Mech. Engrg., 195(52): 7182-7195, 2006. ISSN 00457825. doi: 10.1016/j.cma.2005.05.052.

[6] Igniacio Carol, Andrés Idiart, Carlos López, and Antonio Caballero. Multiaxial behavior of concrete A mesomechanical approach. European Journal of Environmental and Civil Engineering, 11(7-8):907-926, 2007. doi: 10.3166/regc.11.907-926.

[7] E Garboczi. Multiscale Analytical/Numerical Theory of the Diffusivity of Concrete. Adv. Cem. Based Mater., 8(2):77-88, 1998. ISSN 10657355. doi: 10.1016/S1065-7355(98) 00010-8.

[8] M.G.D. Geers, V.G. Kouznetsova, and W.a.M. Brekelmans. Multi-scale computational homogenization: Trends and challenges. Journal of Computational and Applied Mathematics, 234(7):2175-2182, August 2010. ISSN 03770427. doi: 10.1016/j.cam.2009.08.077.

[9] D Gross and T Seeling. Fracture Mechanics: With an Introduction to Micromechanics. Springer, 2001. ISBN 978-3-642-19240-1.

[10] M Hain and P Wriggers. Numerical homogenization of hardened cement paste. Computational Mechanics, 42(2):197-212, 2007. ISSN 0178-7675. doi: 10.1007/s00466-007-0211-9.

[11] M Hain and P Wriggers. Computational homogenization of micro-structural damage due to frost in hardened cement paste. Finite. Elem. Anal. Des., 44(5): 233-244, 2008. ISSN 0168874X. doi: 10.1016/j.finel.2007.11.020.

[12] Z Hashin and S Shtrikman. A variational approach to the theory of the effective magnetic permeability of multiphase materials. Journal of Applied Physics, 33(10): 3125, 1962. ISSN 00218979. doi: 10.1063/1.1728579.

[13] Göran Hedenblad. Moisture permeability of mature concrete, cement mortar and cement paste. PhD thesis, Div of Building Materials LTH, Lund university, 1993.

[14] M Karim and K Krabbenhoft. Extraction of Effective Cement Paste Diffusivities from X-ray Microtomography Scans. Transport in Porous Media, 84(2):371-388, 2011. doi: $10.1007 /$ s11242-009-9506-y. 
[15] S Kim and R K A Al-rub. Meso-scale computational modeling of the plastic-damage response of cementitious composites. Cement and Concrete Research, 41(3):339-358, 2011. ISSN 0008-8846. doi: 10.1016/j.cemconres.2010.12.002.

[16] F Larsson, K Runesson, and F Su. Computational homogenization of uncoupled consolidation in micro-heterogeneous porous media. Int. J. Numer. Anal. Meth. Geomech., 34(14):1431-1458, 2009. ISSN 03639061. doi: 10.1002/nag.862.

[17] M Lutz, P Monteiro, and R Zimmerman. Inhomogeneous interfacial transition zone model for the bulk modulus of mortar. Cement and Concrete Research, 27(7): 1113-1122, 1997. doi: 10.1016/S0008-8846(97)00086-0.

[18] P K Mehta and P J M Monteiro. Concrete: Microstructure, Properties and Materials. McGraw-Hill Professional, 2006. ISBN 0071462899. doi: 10.1036/0071462899.

[19] Christian Miehe, Jörg Schröder, and Jan Schotte. Computational homogenization analysis in finite plasticity Simulation of texture development in polycrystalline materials. Comput. Methods Appl. Mech. Engrg., 171(3-4):387-418, April 1999. ISSN 00457825. doi: 10.1016/S0045-7825(98)00218-7.

[20] A. U. Nilsen and P. J.M. Monteiro. Concrete: A three phase material. Cement and Concrete Research, 23(1):147-151, January 1993. doi: 10.1016/0008-8846(93)90145-Y.

[21] J P Ollivier, J C Maso, and B Bourdette. Interfacial transition zone in concrete. Advanced Cement Based Materials, 2(1):30-38, January 1995. ISSN 10657355. doi: 10.1016/1065-7355(95)90037-3.

[22] Martin Ostoja-Starzewski. Material spatial randomness: From statistical to representative volume element. Probabilistic Engineering Mechanics, 21(2):112-132, April 2006. ISSN 02668920. doi: 10.1016/j.probengmech.2005.07.007.

[23] M Promentilla, T Sugiyama, T Hitomi, and N Takeda. Quantification of tortuosity in hardened cement pastes using synchrotron-based X-ray computed microtomography. Cement and Concrete Research, 39(6):548-557, 2009. doi: 10.1016/j.cemconres.2009.03.005.

[24] I Özdemir, W Brekelmans, and M Geers. $\mathrm{FE}^{2}$ computational homogenization for the thermo-mechanical analysis of heterogeneous solids. Comput. Methods Appl. Mech. Engrg., 198(3-4):602-613, 2008. ISSN 00457825. doi: 10.1016/j.cma.2008.09.008.

[25] E. Schlangen and J. G. M. Mier. Simple lattice model for numerical simulation of fracture of concrete materials and structures. Materials and Structures, 25(9):534-542, November 1992. ISSN 0025-5432. doi: 10.1007/BF02472449.

[26] D Sohn, Y.-S. Cho, and S Im. A novel scheme to generate meshes with hexahedral elements and poly-pyramid elements: The carving technique. Comput. Methods Appl. Mech. Engrg., 201-204:208-227, January 2012. ISSN 00457825. doi: 10.1016/j. cma.2011.09.002. 
[27] E. Stora, Q.-C. He, and B. Bary. A mixed composite spheres assemblage model for the transport properties of random heterogeneous materials with high contrasts. Journal of Applied Physics, 100(8):7, 2006. ISSN 00218979. doi: 10.1063/1.2360155.

[28] L Wang and T Ueda. Mesoscale modeling of water penetration into concrete by capillary absorption. Ocean Engineering, 38(4):519-528, 2011. ISSN 00298018. doi: 10.1016/j.oceaneng.2010.12.019.

[29] P Wriggers and S O Moftah. Mesoscale models for concrete : Homogenisation and damage behaviour. Finite Elements in Analysis and Design, 42:623 - 636, 2006. doi: 10.1016/j.finel.2005.11.008.

[30] C Yang and J Su. Approximate migration coefficient of interfacial transition zone and the effect of aggregate content on the migration coefficient of mortar. Cement and Concrete Research, 32(10):1559-1565, 2002. ISSN 00088846. doi: 10.1016/ S0008-8846(02)00832-3.

[31] Z Yuan and J Fish. Toward realization of computational homogenization in practice. Int. J. Numer. Meth. Eng., 73(3):361-380, 2008. ISSN 00295981. doi: 10.1002/nme.2074.

[32] T I Zohdi and P Wriggers. Introduction to Computational Micromechanics. Springer, Berlin, 2005. ISBN 978-3-540-77482-2. 